\title{
Resolução de problemas de estrutura aditiva: a compreensão de uma professora de primeira série
}

\section{Additive structure problem solving: a first grade teacher's understanding}

\author{
DOI: $10.46814 / \operatorname{lajdv3n1-036}$
}

Recebimento dos originais: 30/10/2020

Aceitação para publicação: 23/12/2020

\author{
Alex Oleandro Gonçalves \\ Doutor em Educação pela Pontifícia Universidade Católica do Paraná - PUCPR \\ E-mail: clauealex@yahoo.com.br
}

\section{RESUMO}

Este artigo é resultado de um estudo de caso que foi elaborado com o objetivo de investigar nas aulas de matemática elementos da prática de sala de aula que caracterizassem conceitos teóricos referentes ao campo das Estruturas Aditivas, analisando os problemas e atividades propostos pela professora regente com base nos estudos de autores, teóricos e pesquisadores da Teoria dos Campos Conceituais, especificamente, o campo das Estruturas Aditivas. Esta teoria foi proposta por Gerard Vergnaud, à qual permite compreender melhor como o aluno aprende conceitos matemáticos referentes às operações de adição e subtração. Foram realizadas quatro sessões de observação em uma turma de numa escola pública da região metropolitana de Curitiba, durante os meses de abril e agosto do ano de 2007, em dias combinados com a professora regente, nos quais realizaria o trabalho de resolução de problemas de estrutura aditiva. Trata-se de uma primeira série do ensino fundamental ou segundo ano do atual regime de nove anos, com 27 alunos. Dessas quatro sessões de atividades, foram selecionadas duas que foram consideradas mais relevantes para o estudo. Algumas perguntas abertas foram feitas à professora regente com o intuito de esclarecer alguns pontos da observação. Os resultados apontam para o fato de que a prática docente com relação à resolução de problemas de estrutura aditiva está fundamentada, basicamente na troca de experiência entre professores de uma mesma escola, às vezes, sem muita reflexão. Outro aspecto indica que a professora desconhece a Teoria dos Campos Conceituais e trabalha isoladamente cada operação, priorizando o cálculo escrito sem estabelecer relação entre estas operações.

Palavras-chave: Adição, Subtração, Estruturas Aditivas, Campos Conceituais, Educação Matemática.

\begin{abstract}
This article is the result of a case study that was developed with the objective of investigating in mathematics classes elements of classroom practice that characterize theoretical concepts related to the Additive Structures field, analyzing the problems and activities proposed by the regular teacher based on the studies of authors, theorists and researchers of the Conceptual Fields Theory, specifically, the Additive Structures field. This theory was proposed by Gerard Vergnaud, which allows a better understanding of how the student learns mathematical concepts related to addition and subtraction operations. Four observation sessions were conducted in a public school class in the metropolitan area of Curitiba, during the months of April and August of 2007, on days agreed with the regular teacher, when the work of solving additive structure problems would take place. This is a first grade of elementary school, or second year of the current nine-year regime, with 27 students. Of these four activity sessions, two were selected as most relevant for the study. The teacher was asked some open questions in order to clarify some points of the observation. The results point to the fact that the teaching practice regarding the resolution of additive structure problems is basically based on the
\end{abstract}


exchange of experiences among teachers of the same school, sometimes without much reflection. Another aspect indicates that the teacher is unaware of the Conceptual Fields Theory and works each operation separately, prioritizing the written calculation without establishing a relationship between these operations.

Keywords: Addition, Subtraction, Additive Structures, Conceptual Fields, Mathematics Education.

\section{INTRODUÇÃO}

A Teoria dos Campos Conceituais, proposta por Vergnaud (1983, in: MAGINA, 2001), oferece um referencial ao estudo do desenvolvimento cognitivo e da aprendizagem de competências complexas, particularmente àquelas implicadas nas ciências, levando em conta os próprios conteúdos do conhecimento e a análise conceitual de seu domínio. De acordo com essa teoria, a compreensão de um conceito, por mais simples que seja, não emerge apenas de um tipo de situação, assim como uma simples situação sempre envolve mais do que um único conceito. É levada em conta uma série de fatores que influenciam e interferem na formação e no desenvolvimento de conceitos, segundo a Teoria dos Campos Conceituais. Ainda segundo essa teoria, o conhecimento conceitual deve emergir dentro de situações problema.

\subsection{O CAMPO CONCEITUAL ADITIVO}

É comum entre professores das séries iniciais achar que problemas de adição devem ser ensinados antes dos problemas de subtração por serem considerados mais fáceis. Os Parâmetros Curriculares Nacionais ressaltam que a dificuldade de um problema não está relacionada, necessariamente, à operação requisitada para sua solução: "Isso evidencia que os problemas não se classificam em função unicamente das operações a eles relacionadas a priori, e sim em função dos procedimentos utilizados por quem os soluciona" (BRASIL, 1997).

No que diz respeito ao Campo Conceitual das Estruturas Aditivas (VERGNAUD, in: MORO \& SOARES, 2005), muitas são as dificuldades encontradas por professores em todos os níveis de ensino, pois não tiveram oportunidade de discutir o assunto no curso de formação ou não tiveram bons cursos de atualização para tratar desse assunto. Nesse sentido, nos deparamos com perguntas dos alunos como “É ‘de mais' ou é ‘de menos' professora?” e com situações em que os professores acabam facilitando para que os alunos sejam capazes de resolver problemas em que, muitas vezes, os únicos conceitos trabalhados são o de "tirar”, na subtração, e o de “juntar”, na adição.

Os problemas de estrutura aditiva classificam-se, conforme a nomenclatura utilizada, por sua característica em: problemas de transformação, de comparação e de composição. Um dos primeiros 
problemas que a criança domina, aos 5-6 anos, é o de composição (Figura 1), em que estão envolvidas as partes para formar o todo.

Figura 1: Modelo do diagrama para problemas de composição.

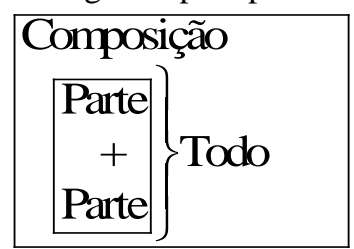

FONTE: Magina (2001)

O tipo de problema mais comum é conhecido como de transformação (Figura 2), em que a quantidade inicial é transformada por uma ação de ganho ou perda - ganhar, perder, tirar, aumentar, diminuir, dar, receber, etc. - e, geralmente uma pergunta pede a quantidade final - Quanto ficou? Quanto restou? Quanto tem agora? etc.

FIGURA 2: Modelo do diagrama para problemas de transformação.

\begin{tabular}{|c|c|}
\hline \multicolumn{2}{|l|}{ Transformação } \\
\hline Estado Inicial & Ação $\rightarrow$ Estado Final \\
\hline & $\begin{array}{l}\text { ganho ou } \\
\text { perda }\end{array}$ \\
\hline
\end{tabular}

FONTE: Magina (2001).

Esse tipo de problema, juntamente com os primeiros de composição são os protótipos da estrutura aditiva. Problemas de transformação em que se pede a quantidade inicial ou a transformação; problemas de composição em que se pede uma das partes e os problemas de comparação e suas variações são chamados de extensões. Problemas que envolvem mais que um tipo de raciocínio aditivo simultaneamente são mistos.

Problemas de comparação (Figura 3) levam os alunos a desenvolverem esquemas de ação mais elaborados para resolvê-los, pois nem sempre fica evidente a operação a ser realizada.

Figura 3: Modelo do diagrama para problemas de comparação.

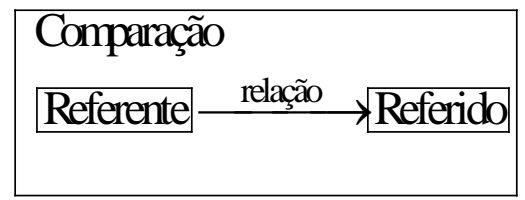

FONTE: Magina (2001).

De acordo com a Teoria dos Campos Conceituais, cada conceito pode ser inserido em um campo conceitual, um conjunto de situações cuja apropriação requer o domínio de vários conceitos, 
desenvolvidos durante um longo período de tempo por meio da experiência, maturação e aprendizagem. Problemas que envolvem as operações de adição e subtração devem ser trabalhados durante todo o ensino fundamental, pois a competência para resolver problemas aditivos é desenvolvida num longo período de tempo.

Segundo Piaget (in: MAGINA, 2001), as crianças desenvolvem os esquemas de juntar e separar independentemente um do outro, sem compreender a relação que existe entre os dois. Para atingir uma compreensão mais avançada, passando do conhecimento baseado em esquemas de ação para um conceito operatório de adição e subtração, é necessário que o aluno consiga coordenar os dois esquemas reconhecendo a relação inversa que existe entre os dois.

Dentro dessa perspectiva, não se pode atribuir uma separação entre o que é um problema de mais, ou o que é um problema de menos. Cada problema de estrutura aditiva é classificado segundo o tipo de raciocínio empregado em sua resolução e a representação matemática dessa resolução depende de como o problema foi interpretado. Assim, o papel determinante do ensino da matemática é procurar meios para estabelecer a relação entre os conceitos matemáticos e a resolução de problemas, os quais dependem da estrutura, do contexto, da característica numérica dos dados, e da apresentação.

A seguir, descrevemos os resultados da investigação feita numa primeira série analisando-se as atividades e problemas observados com o enfoque nas estruturas aditivas.

\section{O ESTUDO REALIZADO}

Foram observadas quatro aulas de matemática de uma primeira série - ou segundo ano no regime de nove anos - com 27 alunos, entre os meses de abril a agosto em uma escola municipal de Quatro Barras / PR, procurando estabelecer uma relação entre a prática de sala de aula e a teoria. Dessas observações realizadas, foram selecionadas para comentário e reflexão duas das atividades consideradas relevantes para o estudo das relações entre a prática de sala de aula e a teoria dos campos conceituais, especificamente do campo conceitual aditivo.

\subsection{A PRIMEIRA ATIVIDADE}

Em uma das primeiras aulas observadas, no início do mês de abril, a professora propôs uma atividade, segundo ela, complementar à do livro didático, de dez exercícios em que era necessário: calcular a soma de objetos, contando as partes e registrando o todo e calcular o total de pontos nos dominós (Figura 4). 
Figura 4: Modelo de exercício aplicado na aula.

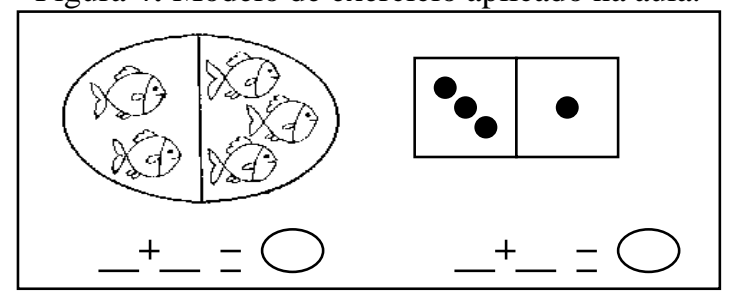

A atividade, da forma como está apresentada tem, como único objetivo, desenvolver a contagem, pois se trata de um problema de composição, em que são dadas as partes e o aluno deve dar o todo, contando as imagens.

Durante a execução da atividade, percebeu-se que, mesmo com as figuras para serem contadas, alguns alunos faziam o registro numérico das parcelas e contavam o total nos dedos. Outras, porém, faziam a conservação de uma das quantidades e realizavam contagem na seqüência a partir do ultimo número dado. Com base em experiências realizadas por Nunes e Bryant (1997) com crianças de 5 e 6 anos acredita-se que a estratégia da contagem na seqüência ajuda a compreender melhor a estrutura decimal.

Estudos realizados por Bryant (1987, in: NUNES \& BRYANT, 1997) mostraram que crianças que usam eficientemente essa solução podem não saber contar a operação aritmética que deveriam usar para resolver o problema.

Segundo a professora regente:

"No início do ano letivo, muitas crianças iniciam na primeira série sem saber ao menos contar uma quantidade pequena de objetos".

Convém ressaltar que a contagem não pode ser vista como uma habilidade à parte. $\mathrm{O}$ conceito de número deve ser construído relacionado com a idéia de adição, mesmo que ainda não haja o registro por escrito da situação.

Alguns alunos usavam como referência para registrar o número uma espécie de reta numérica que vai do numero 1 até o número 31, utilizada como marcador do dia do mês, posicionada sobre o quadro. Estes estavam fazendo o que Nunes (2005) chama de correspondência um a um com os rótulos numéricos.

Durante a correção da atividade, a qual fora realizada com a resolução de cada exercício no quadro observou-se que a professora procurou dialogar bastante com os alunos e que a turma foi muito participativa.

O fato de as crianças apresentarem alta taxa de sucesso em alguns problemas de adição e subtração não significa necessariamente que tenham dominado os conceitos de adição. Devem-se 
propor problemas que exijam variados tipos de raciocínio e em variadas situações. Como destacam os autores:

\begin{abstract}
Mas não é suficiente saber apenas que somar aumenta e subtrair diminui o número de elementos. As crianças devem também entender que essas mudanças exercem efeitos inversos - uma cancela a outra: de modo que $5+2-2=5$. Há diversas razões pelas quais a compreensão dessa regra é importante, e uma delas diz respeito ao que é chamado de composição aditiva do número (NUNES \& BRYANT,1997, p.23).
\end{abstract}

A atividade seguinte fora selecionada, entre outras, devido à quantidade de conceitos envolvidos no que diz respeito às estruturas aditivas.

\title{
2.2 A SEGUNDA ATIVIDADE
}

Em observação realizada no final do mês de junho, a professora propôs uma atividade (Figura 5) que deu seqüência a uma aula em que os alunos leram, com o auxílio da professora, um texto poético denominado "A casa e seu dono" (JOSE, 1987), o qual associava por rima alguns animais ao tipo de casa que moravam. A professora entregou uma folha que continha vários desenhos de animais que faziam parte da poesia, reproduzidos em quantidade não maior que 10, arranjados aleatoriamente para contagem e resposta às questões propostas, o que está de acordo com o que propõe Nunes (2005) afirmando que é melhor desenvolver contagem de objetos dispostos aleatoriamente que arranjados em linha reta, para não correr o risco de reduzir a tarefa de contagem a apenas à correspondência termo a termo.

Os problemas 1, 2 e 8 são de comparação baseados em contagem simples e foram respondidos rapidamente pela maioria dos alunos, bem como os problemas 3 e 4 que são de composição. Estes foram facilitados, provavelmente pela presença da palavra total em sua estrutura, associando a uma adição e, além disso, a contagem pôde ser realizada sobre as próprias figuras. No entanto, os problemas 5, 6 e 7, de comparação nos quais se pede para quantificar as diferenças, foram mais trabalhosos. A análise do exercício será concentrada nesses três itens. 
Figura 5: Modelo da atividade aplicada na aula.

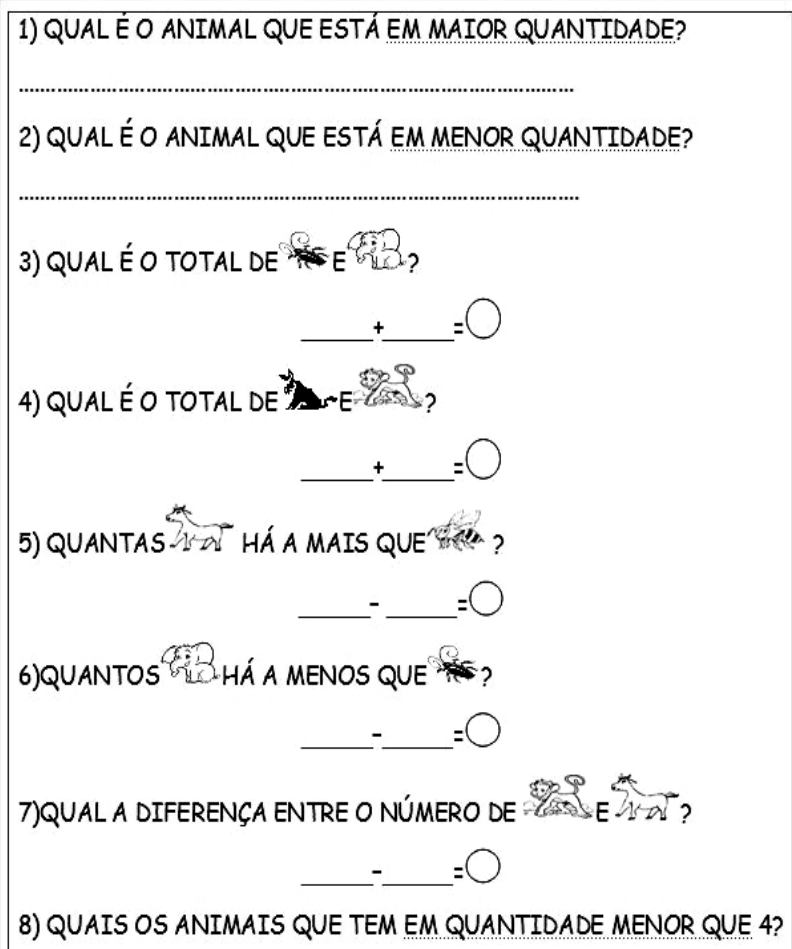

Em problemas de comparação de quantidades, como o caso dos números 5 e 6, o uso de palavras chave pode facilitar ou dificultar o raciocínio para a faixa etária em questão. No problema 5 a palavra mais leva a falsa interpretação de que as quantidades devem ser somadas e, no problema 6, a palavra menos leva diretamente a solução do problema por uma subtração, sem que as crianças façam o uso do raciocínio aditivo para resolvê-lo. Isto foi realmente o observado com a maioria dos alunos da turma. Houve alunos que ignoraram o fato de a professora já ter colocado o registro da operação de subtração e realizaram uma adição devido à presença da expressão a mais que.

Estudos realizados por Moro (in: MORO \& SOARES, 2005) revelam que a ausência de pistas, chamadas por ela de marcas, para que as crianças identifiquem a subtração na situação problema, mostrou o árduo caminho da elaboração dos esquemas subtrativos com cálculo do complemento $-a$ mais que, a menos que - e a importância da intervenção do adulto na construção e elaboração desses esquemas. Nunes (2005) em seu trabalho com esse tipo de problema, antes de fazer a pergunta quanto a menos, pergunta quem tem menos e o equivalente para quanto a mais.

A autora sugere que o professor discuta a solução com as crianças e reformule o problema oralmente. Perguntando, por exemplo, no problema 5, quantas abelhas deveriam chegar para que ficasse a mesma quantidade de abelhas e cabritas, não se trataria mais do mesmo problema. Essa questão se refere a uma transformação e, em geral, segundo a autora, conduz à resposta certa. Esse tipo 
de problema é referido como um problema de equalização justamente porque envolve uma transformação, sendo distinguido das comparações estáticas.

Problemas como os números 5 e 6 são muito mais complexos do que simplesmente, identificar a situação de comparação em expressões como a mais que, a menos que e aplicar a operação subtração: “O número maior menos o menor" como é normalmente ensinado. Estes são difíceis para as crianças mais novas, mas não quer dizer que não devam ser trabalhados, pois de acordo com estudos realizados por Nunes e Bryant, o trabalho com esse tipo de problemas nos revela muito sobre a razão das dificuldades encontradas pela maioria das crianças.

\begin{abstract}
Estabelecer relação entre as situações de comparação e operações aritméticas não é uma questão simples. Em problemas de transformação, em que as coisas são tiradas ou somadas, as crianças podem facilmente descobrir que ações elas precisam efetuar para resolver um problema com o apoio de blocos ou com seus dedos. As ações realizadas com objetos simbólicos são análogas às que seriam realizadas com os próprios objetos. Quando problemas envolvem comparações estáticas, no entanto, a conexão entre a situação e uma operação sobre objetos simbólicos que conduziria à solução do problema não fica imediatamente clara, porque nada é somado ou tirado de qualquer um dos conjuntos (NUNES \& BRYANT, 1997, p.130).
\end{abstract}

O problema número 7, também de comparação, trabalha o conceito de diferença. O desafio para a criança é aprender a quantificar essa diferença. Quando se pede a diferença entre duas quantidades é possível que a criança entenda que a resposta esperada seja apenas de dizer que uma é maior do que a outra.

$\mathrm{Na}$ correção feita pela professora no quadro, esta lançou mão de um artifício interessante para os problemas 5, 6 e7 de comparação: desenhou uma espécie de gráfico para que fosse possível a comparação de maneira mais direta (Figura 6). Percebeu-se que ao refazer as perguntas referentes aos problemas de comparação, as respostas dos alunos fluíam naturalmente, demonstrando a compreensão dos questionamentos feitos pela professora: quanto a mais, quanto a menos, qual a diferença.

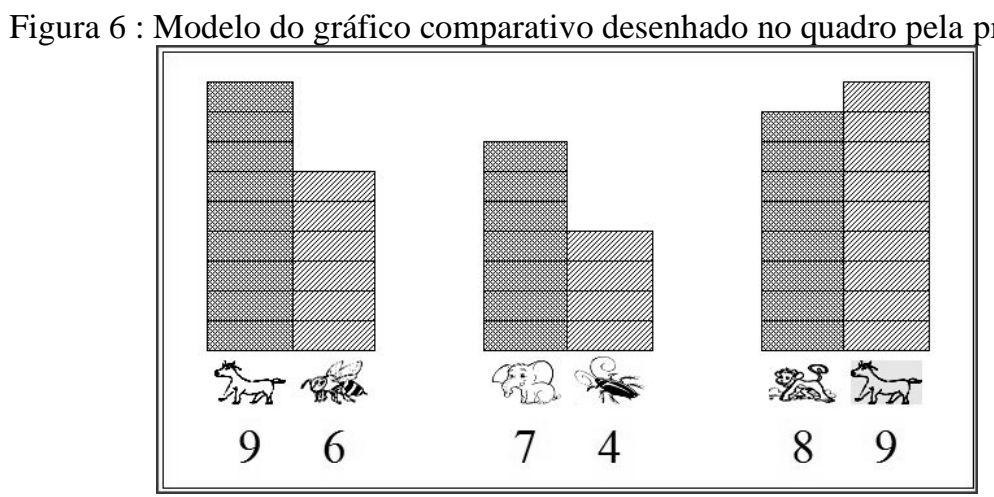

Juntamente com a análise de cada problema durante a correção oral, a professora pediu que os alunos olhassem para a reta numérica posicionada sobre a lousa, a qual era usada como uma espécie 
de calendário para a marcação dos dias do mês, e solicitou que os alunos contassem (em coro, enquanto esta apontava com uma régua) quanto faltava para que as quantidades ficassem iguais.

O procedimento utilizado pela professora para a correção se torna muito útil para ajudar os alunos a desenvolver meios para construir um esquema de ação para problemas de comparação: "Se de uma quantidade maior eu retiro a menor, o que sobra é a diferença entre as duas quantidades". Além disso, ajuda na compreensão da composição aditiva do número; "qualquer número pode ser decomposto em dois outros que vêm antes dele".

Geralmente, o que os alunos usam é a idéia de completar, neste caso, a operação realizada será uma adição. Este raciocínio é válido e pode ser utilizado com sucesso para quantidades pequenas. Segundo Nunes (2005), devem-se propor problemas em que as quantidades exijam utilização de outros meios para resolvê-los. A autora propôs exemplos em que as crianças podem usar a representação gráfica das quantidades para contar a diferença em que se utiliza a reta numérica (Figura 7). Segundo a autora, a utilização de retas numéricas na resolução de problemas faz com que os alunos possam explicitar seu próprio raciocínio e assim, estabelecer a relação com a operação a ser utilizada.

Segundo a autora, após o trabalho com a reta numérica que surge naturalmente no jogo, devese começar a utilizar a reta numérica formal, pois se trata de um instrumento de cálculo e de registro dos alunos. Esta enfatiza que as crianças podem ser incentivadas a reformular problemas de comparação para entender a relação entre as perguntas quanto a mais, quanto a menos, qual a diferença, mudando o referente. No caso do problema 6, em que se pergunta “quantos elefantes há a menos do que baratas?" poderia ser perguntado "quantas baratas há a mais do que elefantes?", entre outras.

Figura 7: Jogo proposto por Nunes.

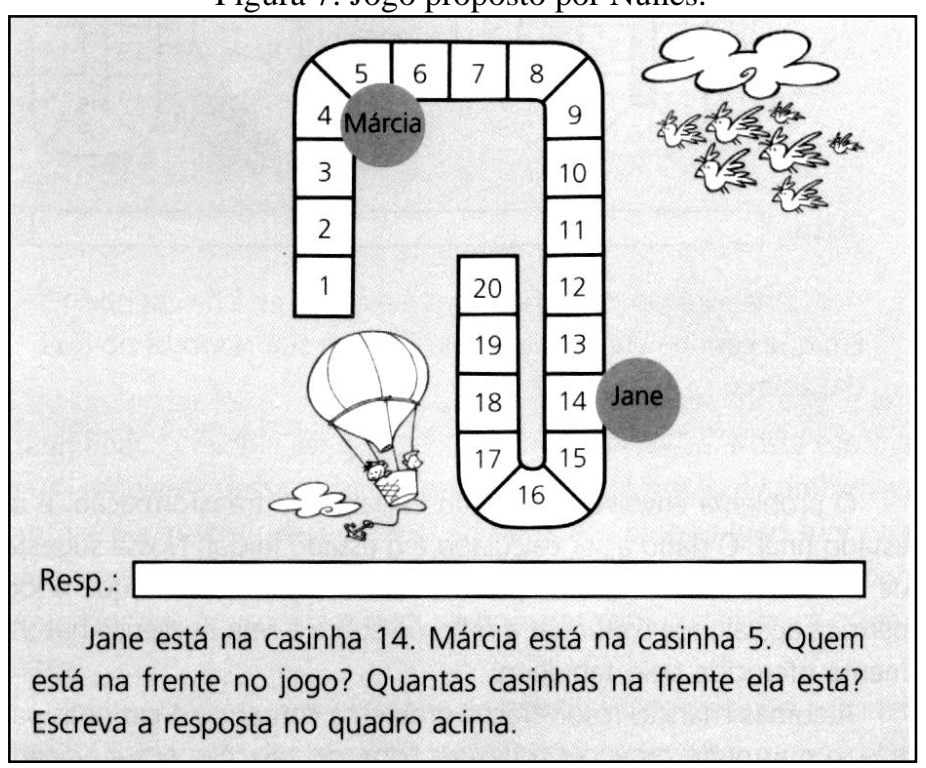

FONTE: Nunes (2005). 
Vergnaud destaca que o sinal de menos tem muitos significados e que nem sempre fica evidente uma subtração pela escrita convencional $(9-5=4)$.

Pensemos também nos múltiplos sentidos do sinal de menos nos raciocínios aritméticos: às vezes uma diminuição, uma perda, um recuo, às vezes uma diferença entre duas quantidades ou duas grandezas, por vezes a inversão de uma transformação (para voltar ao estado inicial, por exemplo), por vezes alguma outra coisa ainda" (VERGNAUD, in: MORO \& SOARES, 2005).

Observou-se, nos problemas propostos, a preocupação da professora em deixar registrada a operação a ser usada com espaços para completar. Essa preocupação com os registros das operações, é chamada por Vergnaud de equívoco do simbolismo da álgebra elementar (in: MORO \& SOARES, 2005). Ainda, segundo ele, o sistema de notação matemática é parte da construção conceitual, mas é algo diferente dessa construção, além de também estar, ele próprio, em construção, porém, as crianças trabalham e devem trabalhar com essas dimensões ao mesmo tempo. O professor tem importante papel no processo de formação e desenvolvimento do raciocínio aditivo, pois deve propor atividades adequadas para criar condições para o aluno aprender significativamente.

\section{CONSIDERAÇÕES FINAIS}

A maioria dos alunos já entra na escola com algum conhecimento acerca do conceito de juntar, tirar e fazer correspondência termo a termo (comparar). O desafio do professor das séries iniciais é desenvolver meios para que o aluno venha a estabelecer relação entre os três esquemas de ação e, portanto, construir um conceito operatório de adição e subtração.

De acordo com a Teoria dos Campos Conceituais, ensinar adição e subtração é muito mais do que apenas ensinar um algoritmo para o cálculo numérico. A criança precisa ser estimulada desde a pré-escola a estabelecer relação entre somar e subtrair, de modo a expandir seu conceito inicial de juntar, tirar e comparar. Problemas de estruturas aditivas não são exclusividade das séries iniciais. $\mathrm{O}$ raciocínio aditivo deverá ser desenvolvido durante todo o Ensino Fundamental.

Pode se perceber através deste trabalho que na turma observada a idéia que as professoras têm de resolução de problemas está vinculada, na maioria das vezes, ao treino de operações matemáticas numa ordem seqüencial - adição, subtração, multiplicação e divisão - e que nenhuma ou pouca relação é estabelecida entre os mecanismos de resolução para cada situação. A resolução de problemas contribui de maneira significativa para o avanço do raciocínio aditivo. Neste sentido, resolver problemas não é simplesmente repetir uma seqüência de procedimentos ensinados pelo professor. O aluno precisa ser incentivado a usar estratégias próprias para a resolução de problemas às quais deverão ser validadas ou não mediante reflexão mediada pelo professor. 
Houve avanços, em relação aos métodos tradicionais de ensino no que diz respeito a aproximar as situações problemas da realidade, ou de situações motivadoras como canções e textos poéticos que fazem parte do universo infantil. No entanto, se faz necessário um trabalho de formação de professores de modo a garantir que os professores conheçam e possam utilizar em seu trabalho os resultados das pesquisas realizadas acerca do campo conceitual aditivo. 


\section{REFERÊNCIAS}

REFERENCIAS

BRASIL, MINISTÉRIO DA EDUCAÇÃO E DO DESPORTO. Parâmetros Curriculares Nacionais - Matemática. Brasília: MEC/SEF,1997.

JOSÉ, Elias. Lua no Brejo. Porto Alegre: Mercado Aberto, 1987.

MAGINA, Sandra et al. Repensando adição e subtração: contribuições de teoria dos campos conceituais. 2. ed. São Paulo: PROEM, 2001.

MORO, Maria F. L.; SOARES, Maria T. C. (Orgs). Desenhos, palavras e números: as marcas da matemática na escola. Curitiba: Ed. da UFPR, 2005.

NUNES, T.; BRYANT, P. Crianças fazendo matemática. Porto Alegre: Artes Médicas, 1997.

NUNES, T.; et al. Educação matemática 1: números e operações numéricas. São Paulo: Cortez, 2005. 